\title{
نحو تعلم تخيلي في مجال تعليمية العربية لغير الناطقين بها
}

Towards an imaginative Learning in the teaching of Arabic as a foreign language

2020/01/19: تاريخ الاستلام :2019/12/05؛ تاريخ القبول

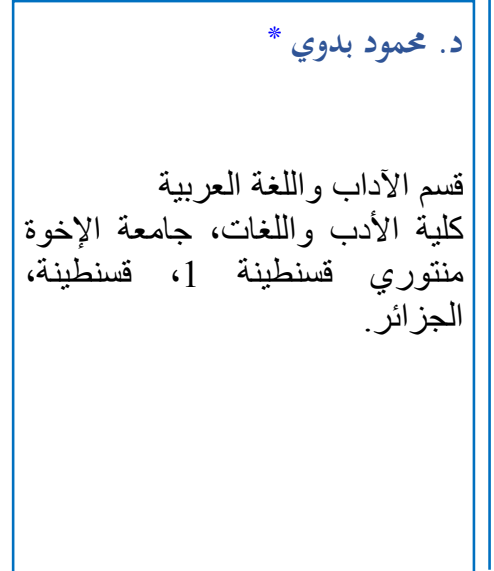

\section{Abstract}

The aim of this article is to suggest how teachers of Arabic as a foreign language might create, through an imaginative and entertaining learning, the use of unusual, funny, mysterious and ambiguous resources, highly inspiring and evocative, a warm and fresh atmosphere where the learner, interacting with humanistic activities speaking to his emotions and childhood remembrances, exciting his curiosity ... dreams, plays with music, conveys odd, strange, unexpected, crazy ... ideas to amaze his classmates and make them laugh, getting rid of that serious and gloomy traditional language classroom!

Keywords: Ambiguous; strange; funny; amaze; unexpected.

\section{Résumé}

Cet article se propose de montrer comment, à travers un apprentissage imaginatif et ludique, des supports didactiques parfois drôles et inédits qui cultivent le gout du flou et de l'étrange, on parvient à créer une classe créative et interactive où l'apprenant d'Arabe, langue étrangère, explorant ses potentialités imaginatives et exprimant son originalité, s'amuse à rechercher les images les plus fantaisistes, insolites et saugrenues pour surprendre ses camarades et déclencher leur fou rire.

Fasciné par un discours caméléon, toujours changeant et surprenant, évocateur et pittoresque, tantôt drôle et amusant, tantôt émouvant et pathétique, tantôt mystérieux et énigmatique ... cet apprenant se libère de la classe de langue traditionnelle, sérieuse et obséquieuse, pour se trouver dans un univers féerique et fantasmatique qui l'inspire et le recrée.

Mots clés: Originalité ; surprise ; drôle ; mystérieux ; imaginatif

* Corresponding author, e-mail: badaoui.mahmoud25@hotmail.com 


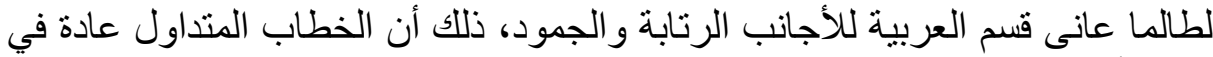

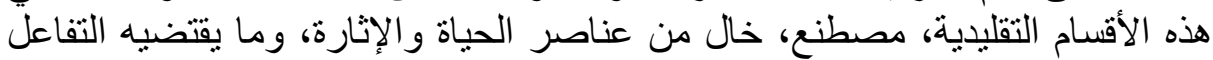

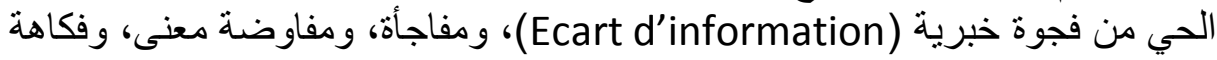

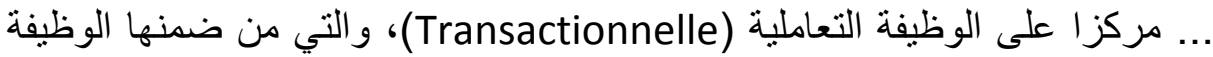
الميتالغوية (Métalinguistique) المنشغلة بتحليل النظام اللغوي، متناسيا أهم وظائفه التفاعلية مثل وظيفة اللعب، ووظيفة التسلية، و الممازحة، و الوظيفة التعبيرية،

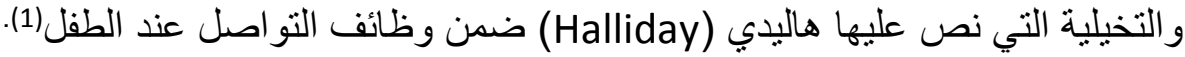
يقول ميشال بوارون (Michel Boiron) بشأن وظيفتي اللعب و والتسلية في مجال

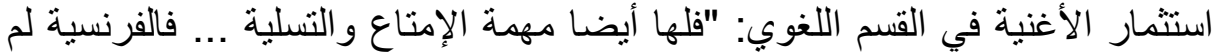

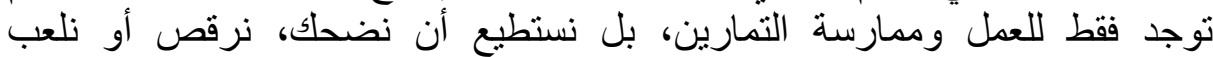

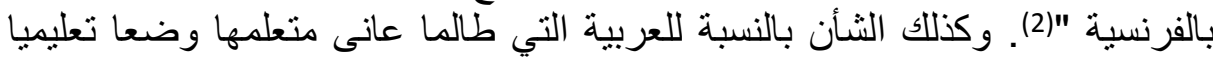

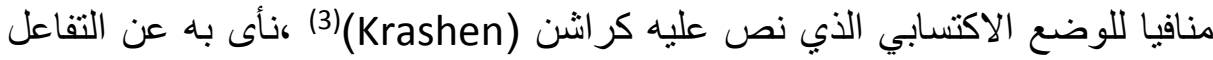

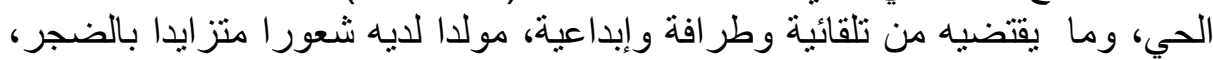

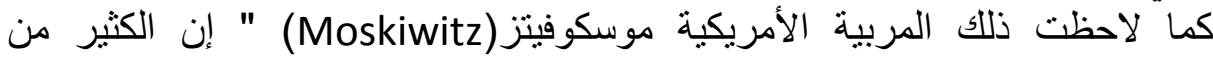
المؤسسات التعليمية التي تعتمد الاستظهار والطرق التقاكية لتقليدية في التعلم والتعليم تجهل الخيال، و لذا فهي جد كئيبة لأنها ليست متخديلة الانيلة "(4). وكرد فعل لهذا الوضع اللاإنساني ظهرت لأنهات في بداية السبعينيات المقاربة الإنسانية (L'approche Humaniste)

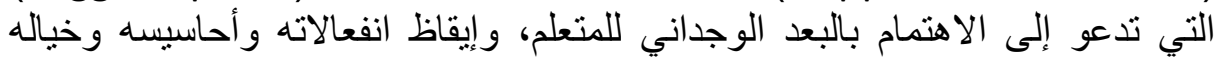

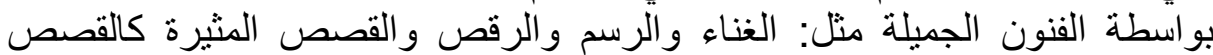

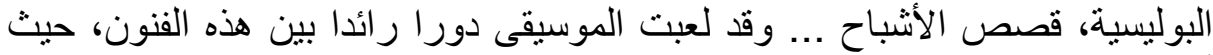

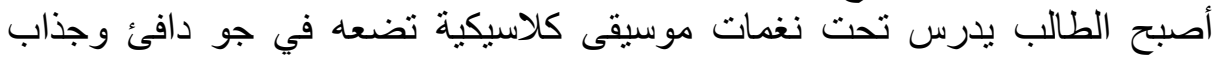

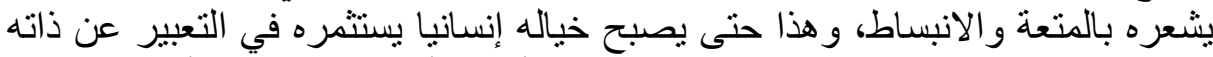

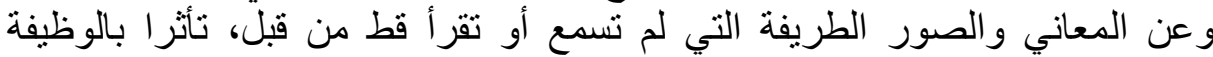

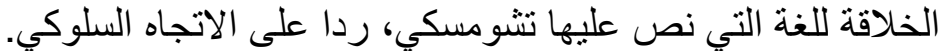

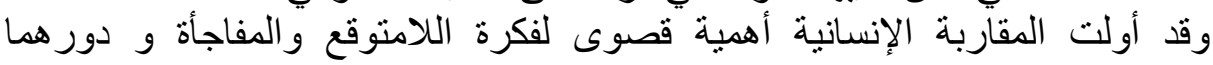

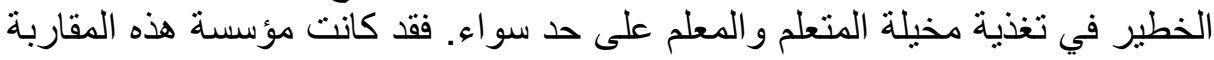

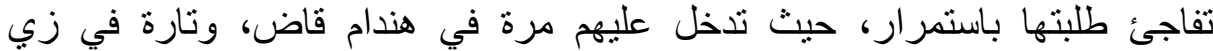

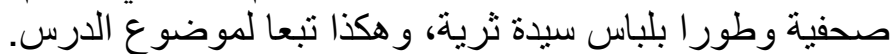

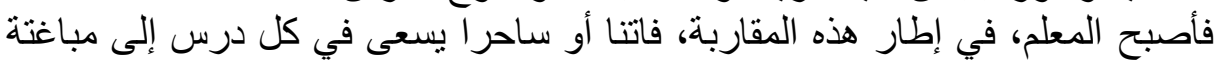

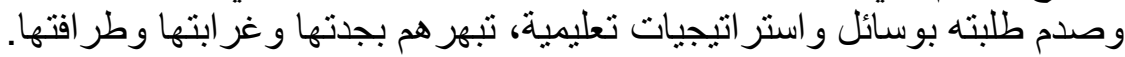

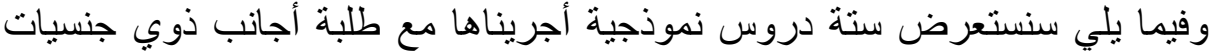

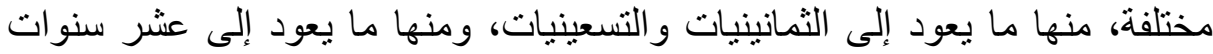

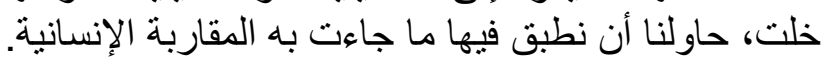

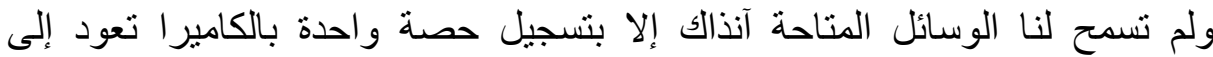

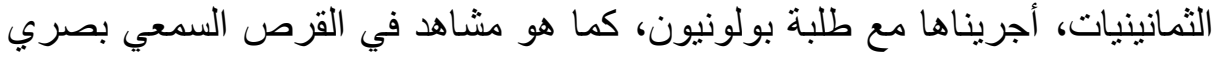

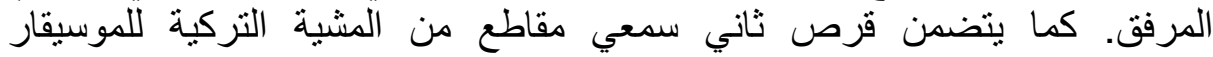

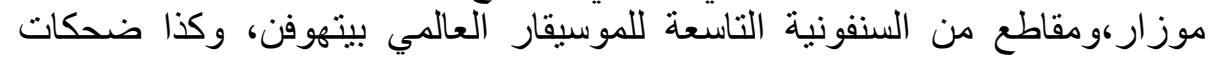
و عطسات قريبة من تللك التي استثمرناها ماضيا. 

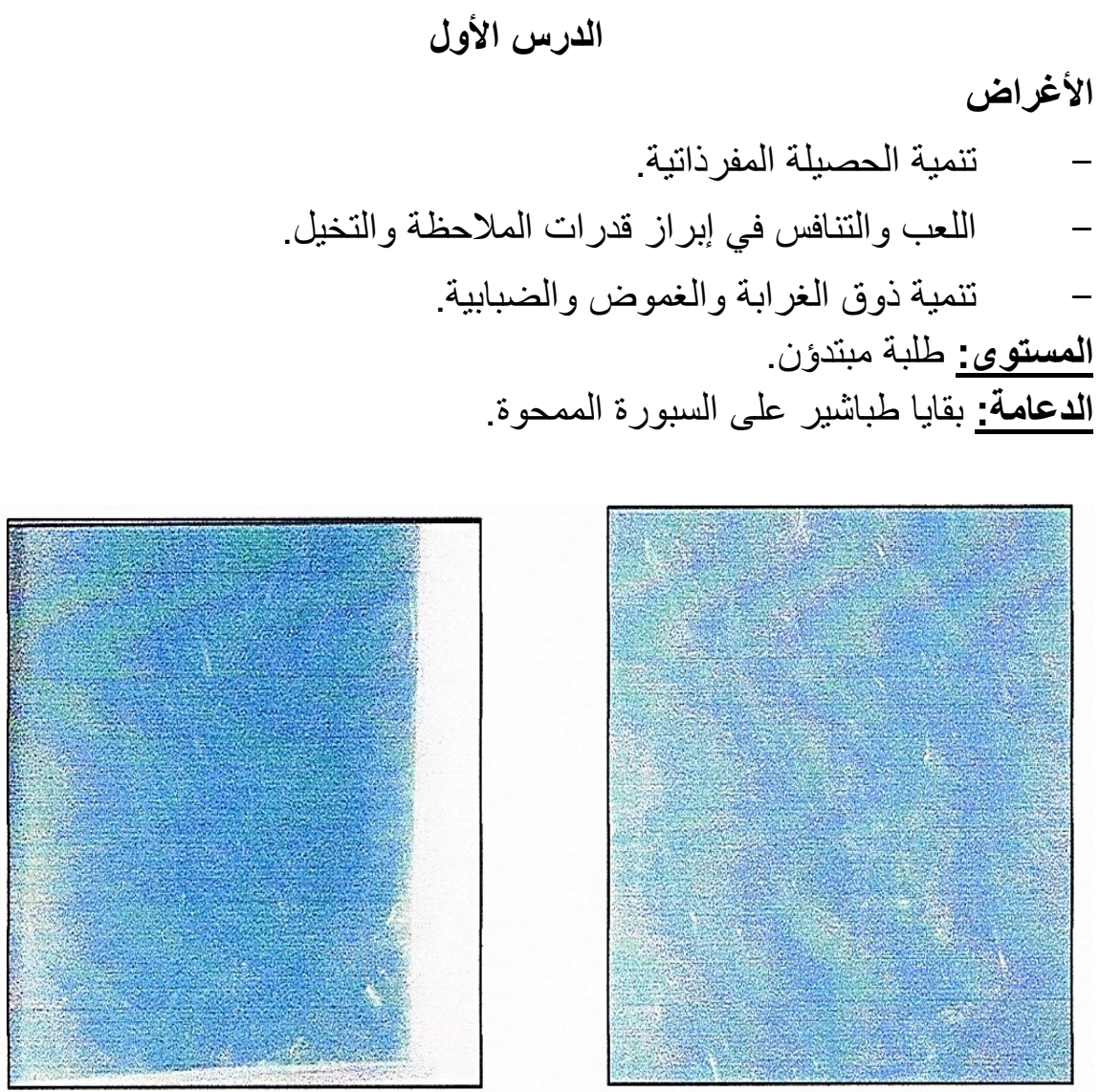

المهمة: يتأمل الطلبة مليا الآثار ثم كلما تصور الطالب شيئا قام إلى السبورة ورسمه.

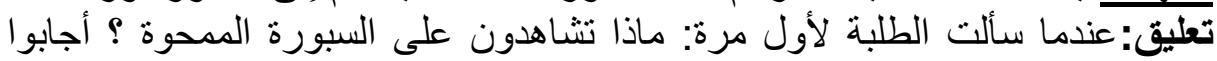

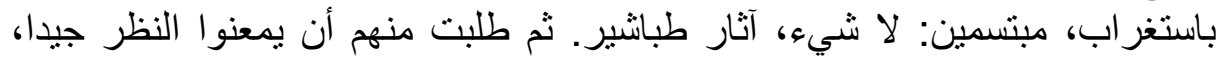

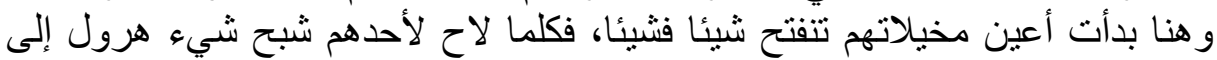

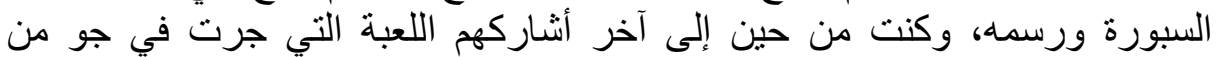
الضحك والمرح. فقد أبدى الطلبة تلهفا في التعبير عن ذكائهم وقدر اتهم الاكتشافية

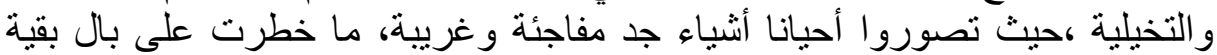
القسم: فقد وجد الطلبة أن بقايا الطباشير نشباء أنشاء

جورب أو قطعة باذنجان

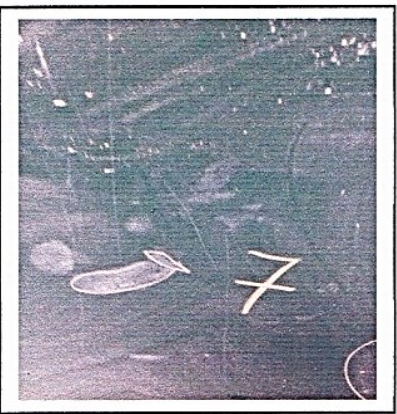

سكة

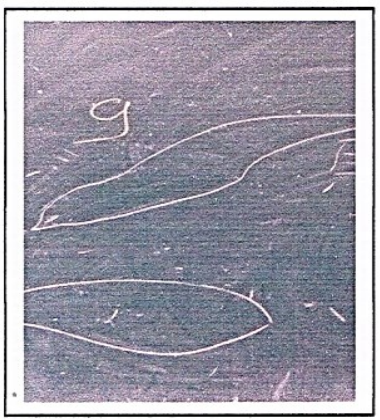

قفاز

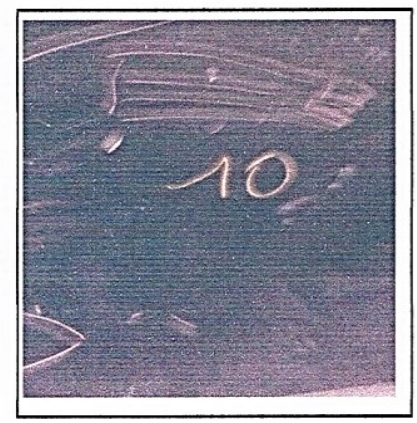




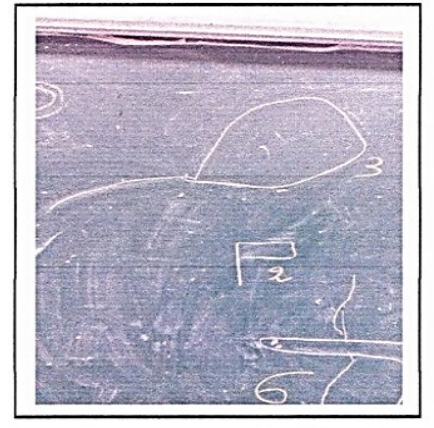

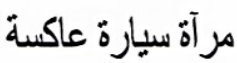
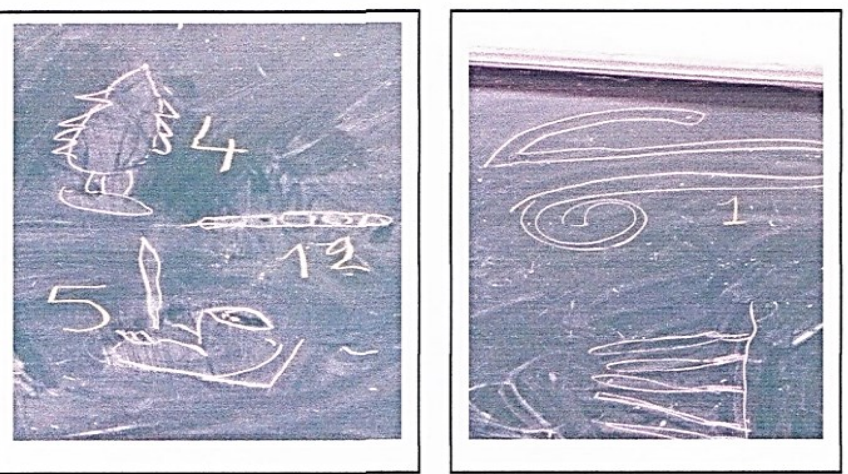

حية

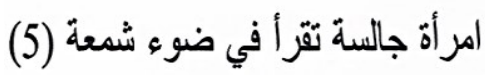

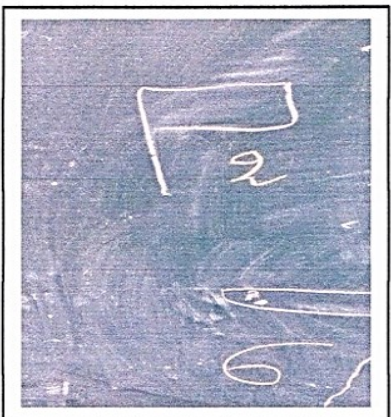

علم
فز اشثة

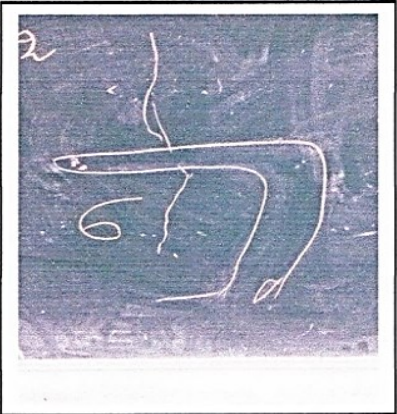

رجل يقوم بحركات رياضية

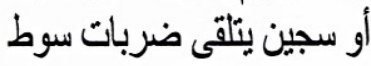

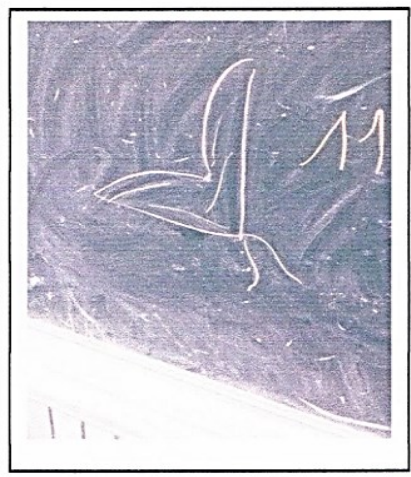

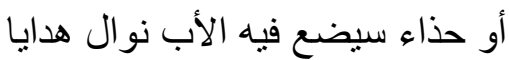

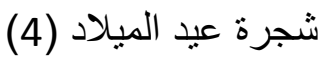

جزء من عمود فقري (12)

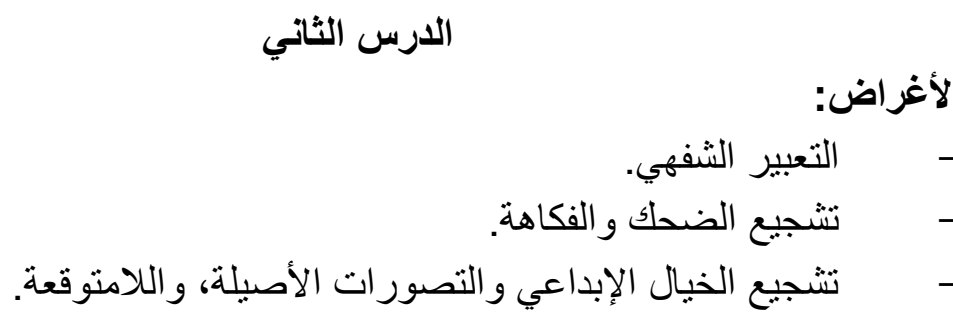

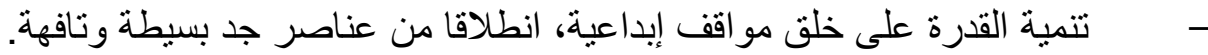

المستوى: ابتدائي وما قبل المتوسط. الاعامة: ضحكات و عطسات طريفة مسجلة.

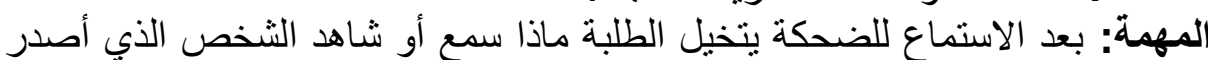

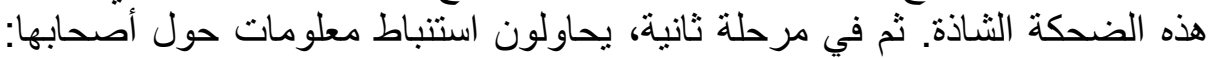

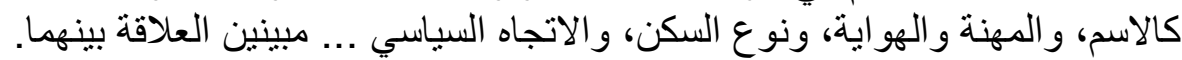

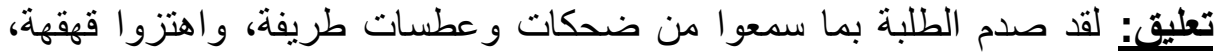
حيث كانوا يتوقعون أنغام أغنية أو مقطو عة موسيقية كما تعودوا. 
ومن بين التخيلات الطريفة التي مازلت أذكرها:

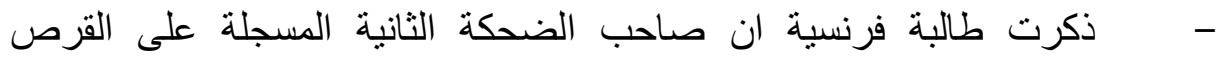

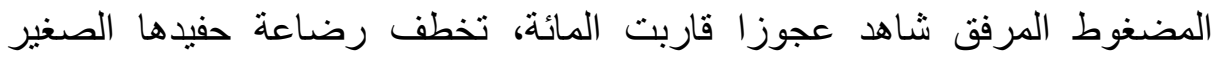

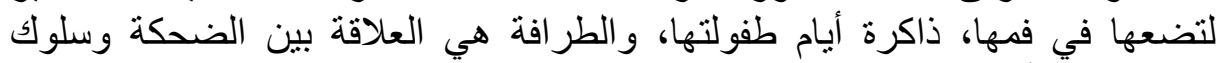

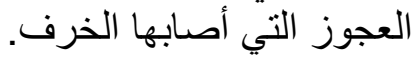

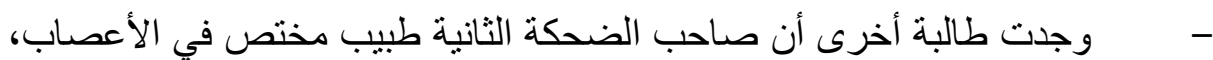

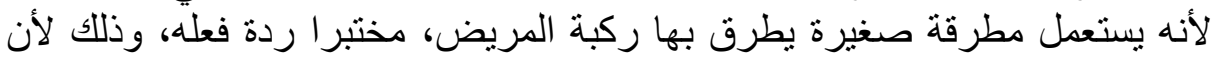

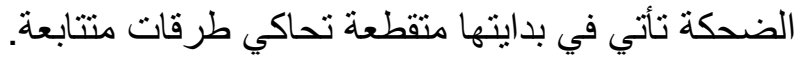

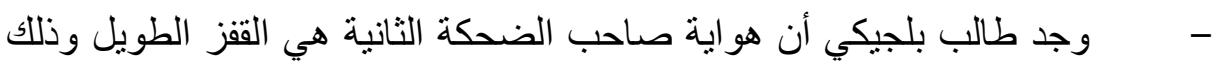
أن الجزء الأول من الضحكة متقطع وسريع يحاكي القفزات الأولى ثلى ثم ينتهي ممددا، يحاكي القزة الأخيرة و الطويلة.

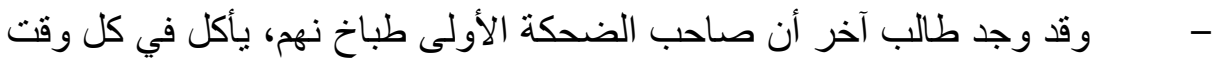

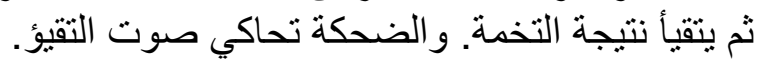

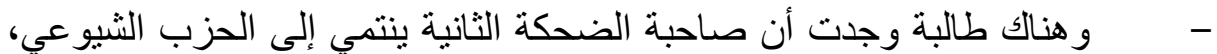
حيث أن أصحابه غلاظ، يضربون على على الطاولة عند عندما يتكلمون.

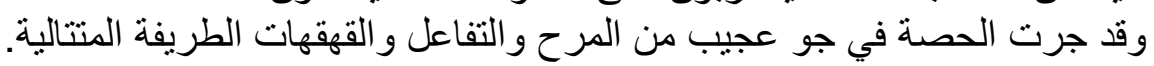

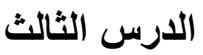

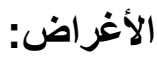

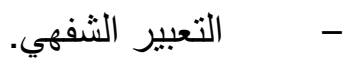

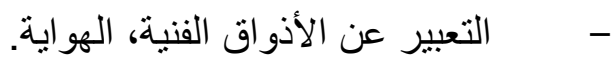

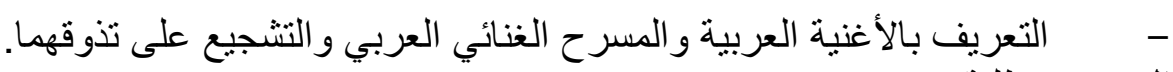
المستوى:طلبة مبتدئون. الدعامة البيداغوجية: مقاطع من سنفونية " كارمن " (G.Brmen) للموسيقار العالمي

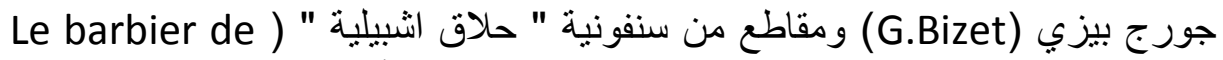
(Séville

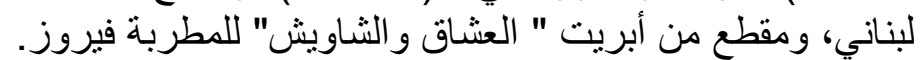

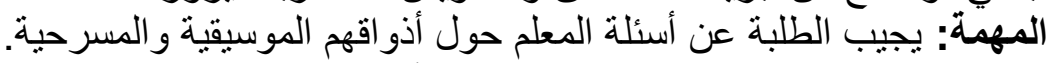
تعليق: بعد الاستماع لمقطوعة " كارمن"، سأل المعلم: هل تحب /تحبين جورج بيزي؟

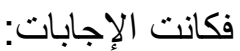

- - معم أنا أحب جورج بيزي ولكن أفضل فريديريك شوبن (F.Chopin). - - معم أنا أحب جورج بيزي ولكن أفضل موريس رافيل (M.Ravel). - - نعم أنا أحب جورج بيزي ولكن أفضل بيتهوفن (Beethoven).

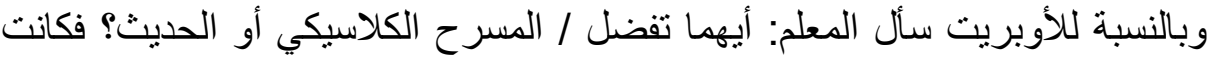

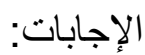

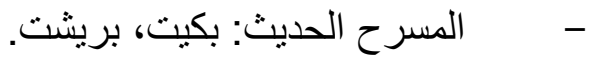

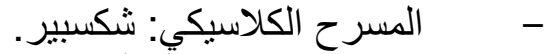

واستطردت طالبة بعفوية: أنا أحب الفنون الجميلة: النحت، الموسيقى، الرسم، السينما. 


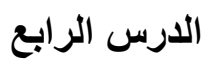

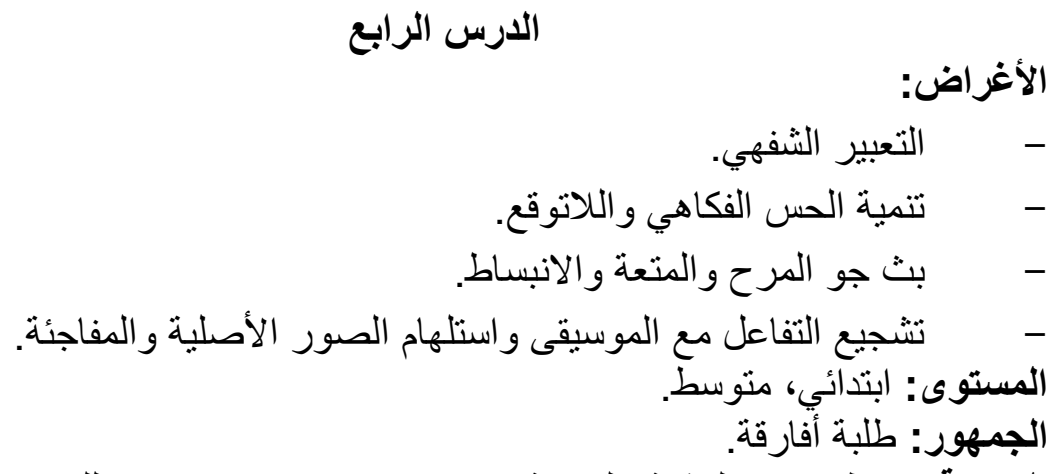

الاعامة: مقطع من المشية التركية (La Marche turque) للموسيقار العالمي

المهمة: استلهام حادثة في منتهى الطر افة و الغر ابة من إيقاعات المقطو عة.

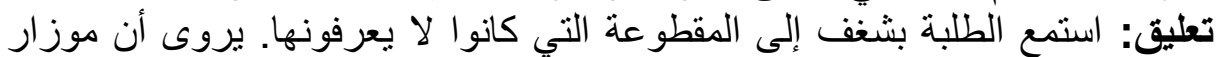

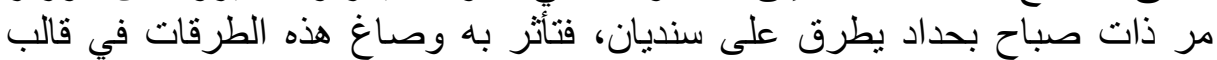

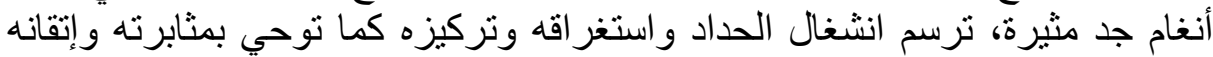

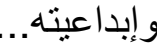
وما زلت أذكر حادثتين طريفتين استوحتهما طالبتان:

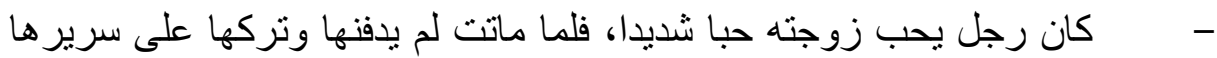

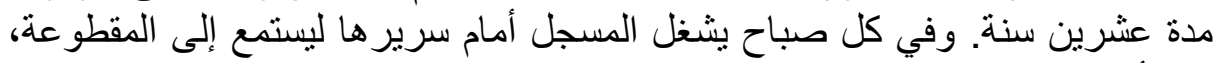

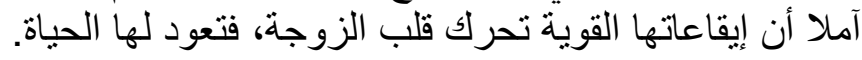

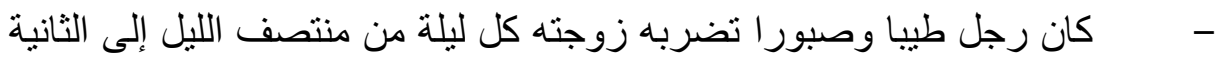

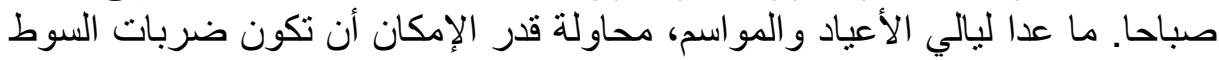

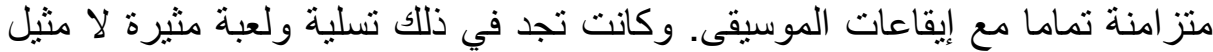

وقد عبر الطلبة عن إعجابهم الثديد ببر اعة خيال الطالبتين، وقهقهو عاليا.

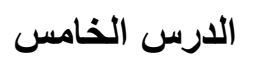

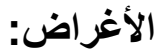

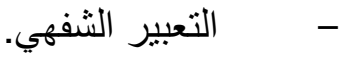

- - مكين الطالب من التعبير عن قدر اته المعرفية: اكتشاف العلاقات، التخيل ... - - - متشيع المفاجئة و الفكاهة.

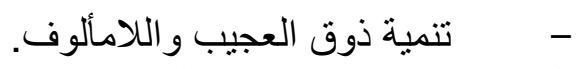
المستوى: مبتدئون ومتوسطون العنون الاعامة: صور مثيرة تعرض وضوض وقائع أقصوصة " اللحية الزرقاء" للكاتب الفرنسي

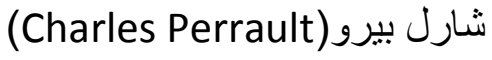


متنطفات من السنفونية الثناسعة للتبوفن.
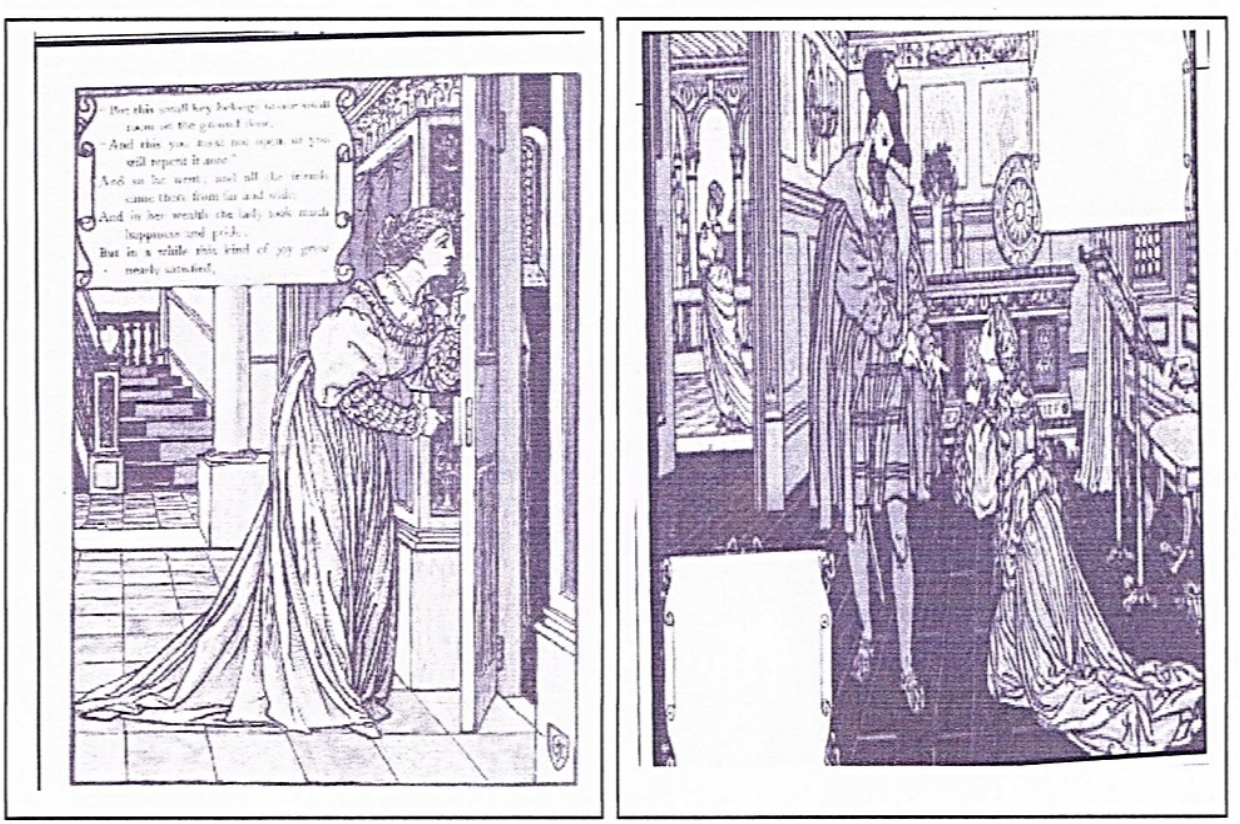

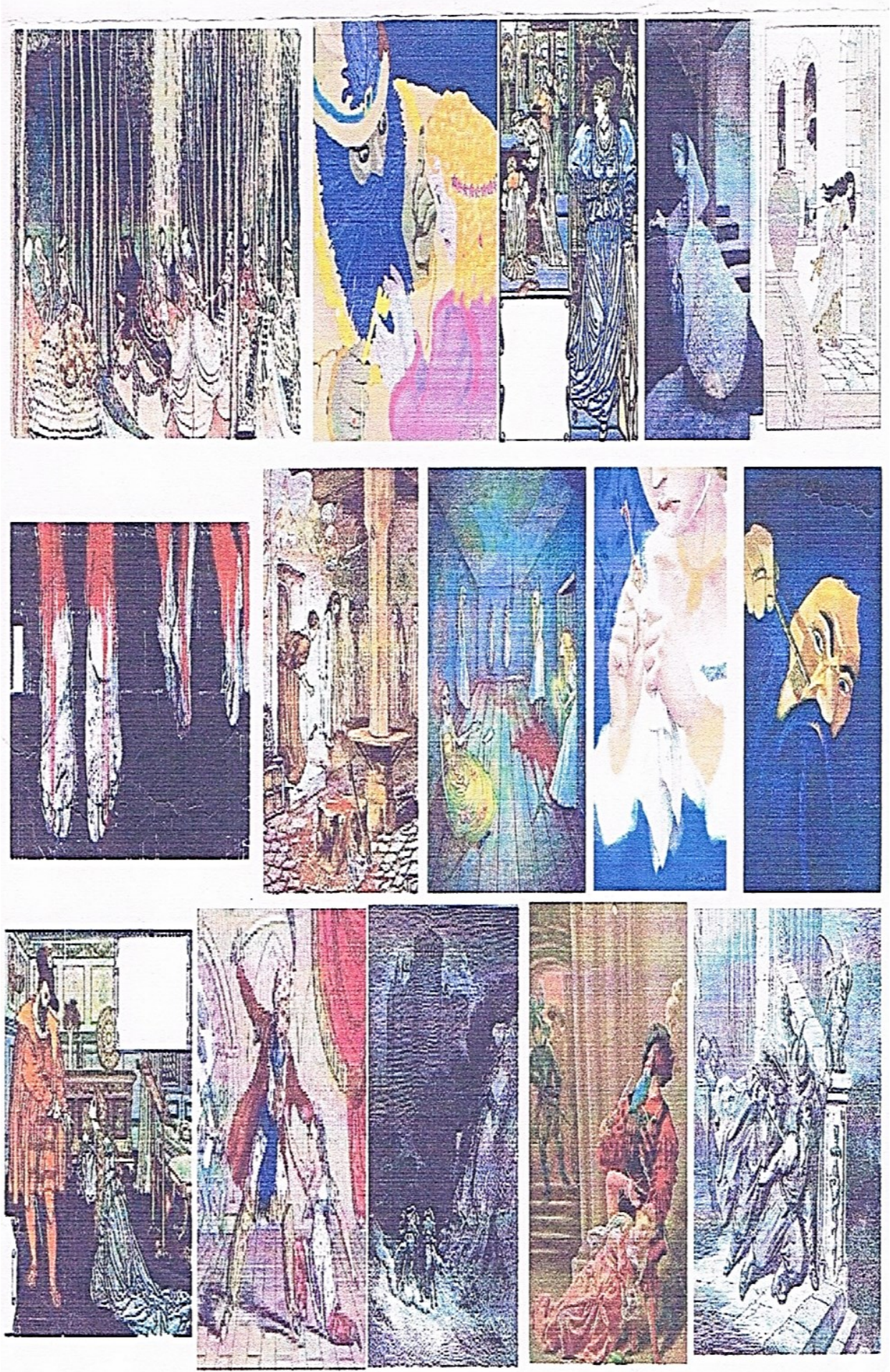

المهمة: بعد التعرف على وقائع الأقصوصة بطريقة تدريجية و مشوقة، تشتثير فضول

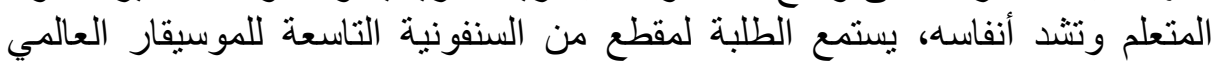
بيتهوفن، ثم يحاولون اكتثاف علاقات بين كل مقطع و الصورة الملائمة. 
تعليق: لقد أوحت الأنغام الثديدة المتتابعة، لبعض الطلبة، مثنا بنسارع دقات قلب

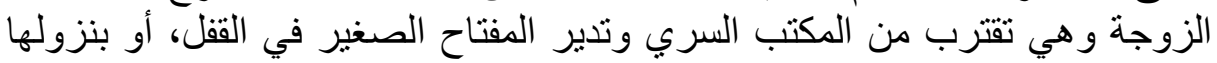

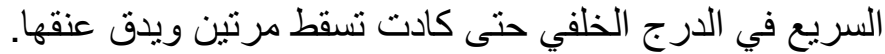

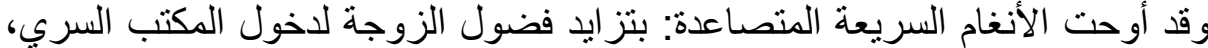

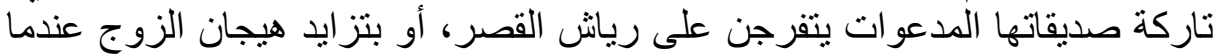

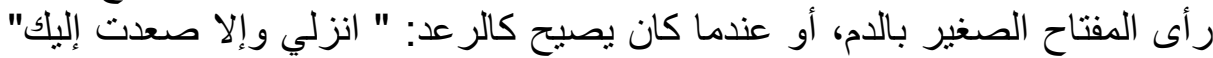

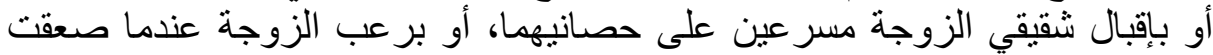
لمشاهدة زوجات اللحية الزرقاء وهن معلقات كالخراف، الواحدة تلو الأخرى، على النى

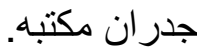
كما أوحت الضربة الثنانية العنيفة بسقوط المفتاح على أرض المكتب المبلة الملطخة بالدماء،

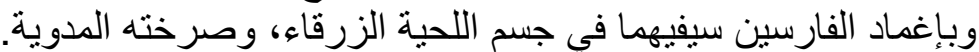
وقد أوحت الأنغام اللطيفة السيالة: بتوسل الزوجة كي لا يقتلها اللحية الزرقاءة، وباءة وبأيام

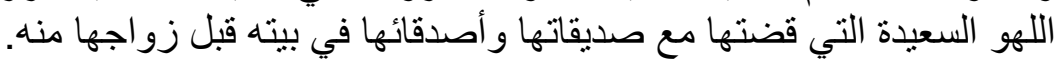

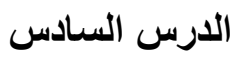

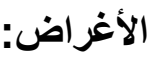

تمية الحصيلة المعجمية.

التعبير الثفهي.

تنمية ذوق الغرابة و المفاجئة، وما يصدم العادي و المألوف.

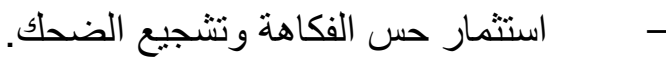

- - موفير جو المتعة والتسلية واللعب.

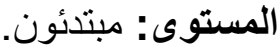

الاعامة: مجمو عة من الصور الطريفة و الهزلية المثيرة للضحك.
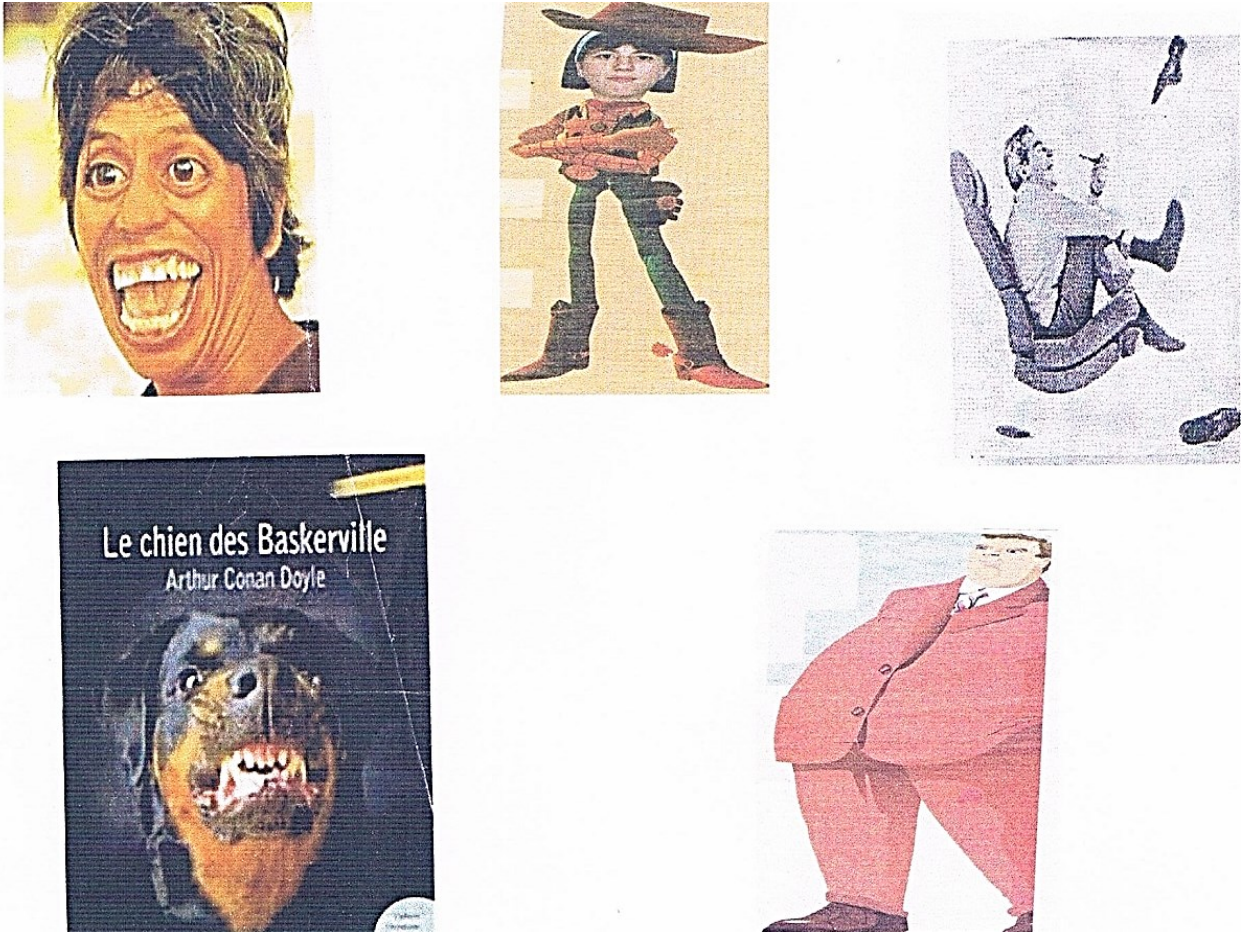
المهمة: يتخيل المتعلم عادة طريفة لأحد هؤلاء الأشخاص، تستثير قهقهة القسم.

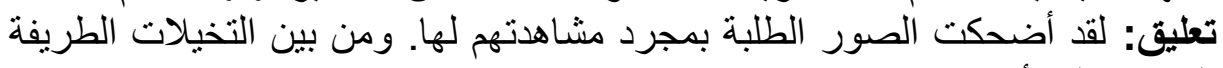
التي مازئ: أت أذكر ها:

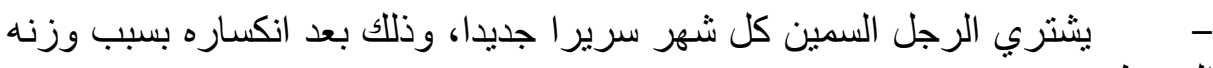
المفرط.

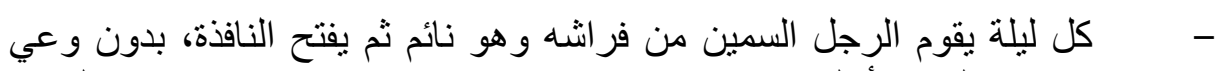

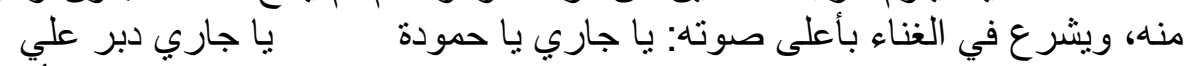

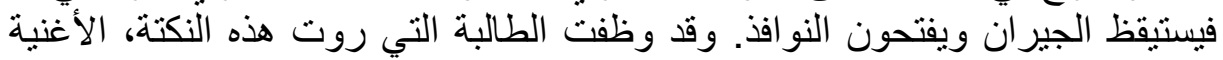

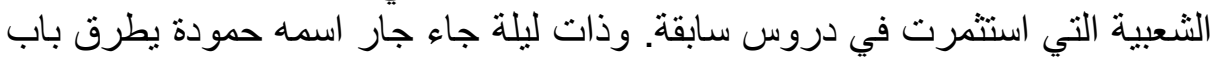
بيته.

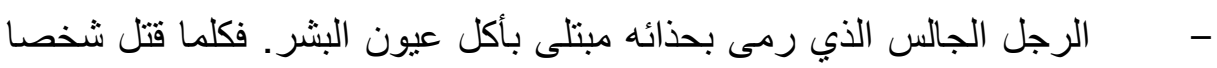

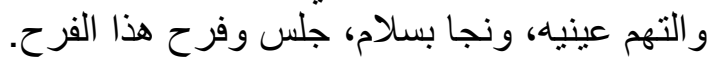

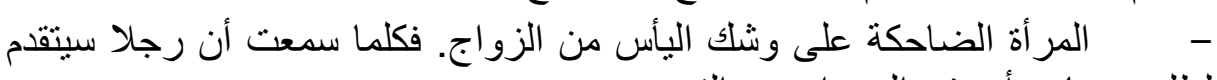

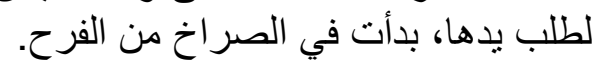
وقد شاركت الطلبة لعبهم فتخيلت رجلا بسكر الكلب ليلة كاملة وذللك قبل أن الن يأمره بقتل ضحيته قائلا: اليوم خمر و غدا أمر ! - - عبر الطلبة عن مواقفت غير جاهزة، كما جرت العادة، و إنما نبعت من مخيلتهم خلال

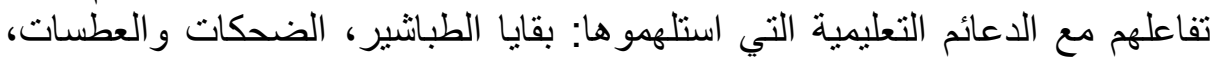

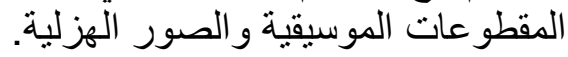

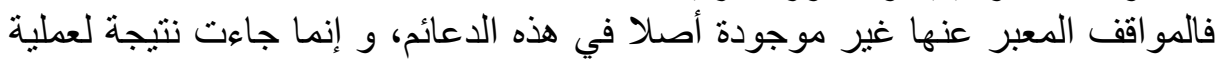

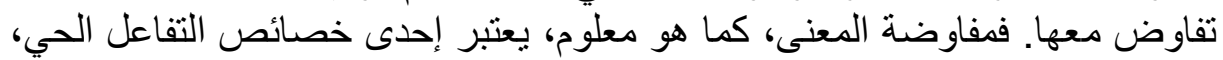

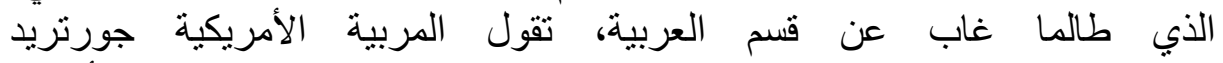

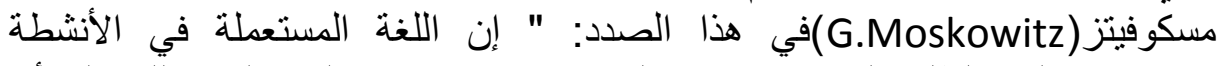

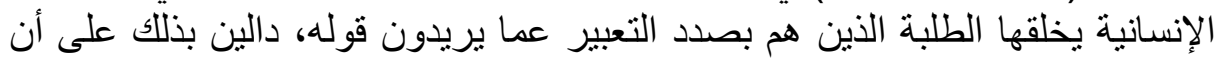

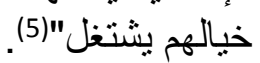

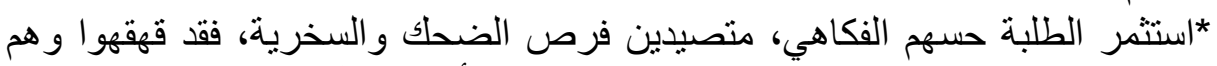

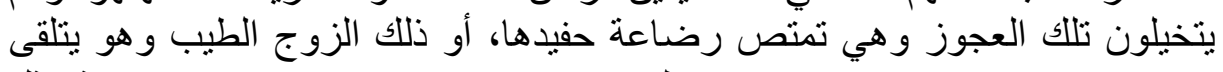

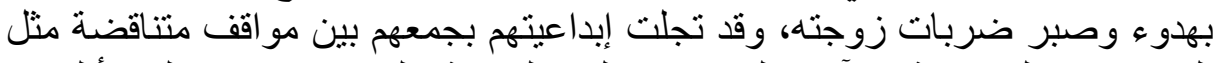

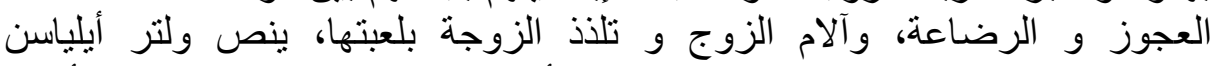
(Walter Eliason) هي في العادة مختلفة"(6).

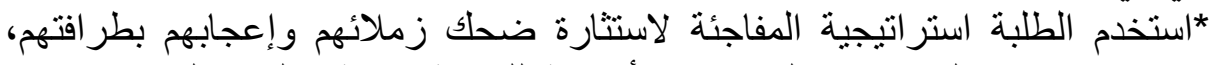

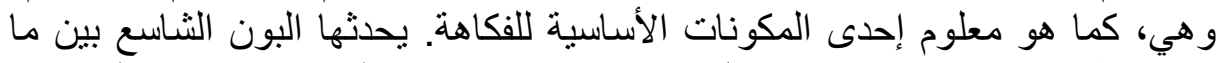

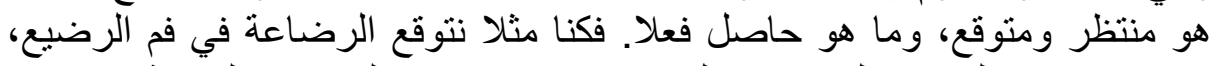

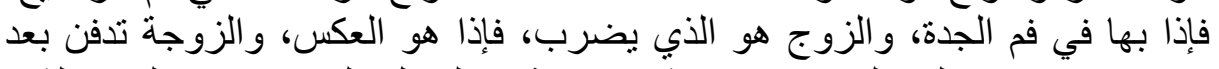

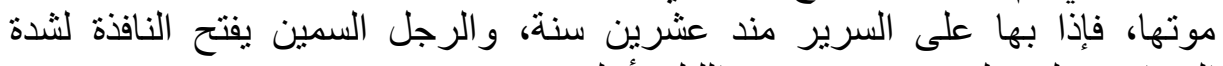

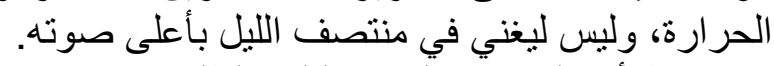

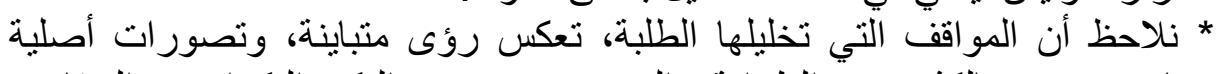

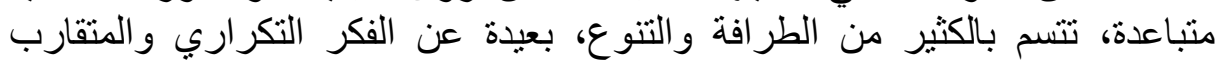


المعهود في الأقسام التقليدية، فقد نص علماء البيداغوجيا أن الأنشطة الإبداعية أصيلة

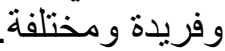
* اكتسب المعلم في إطار المقاربة الإنسانية أو الإبداعية أدوارا جديدة لم تعهدها

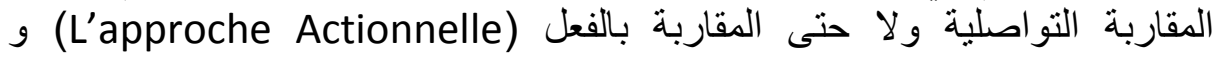

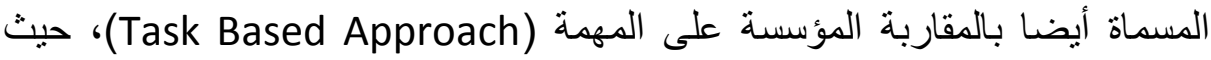

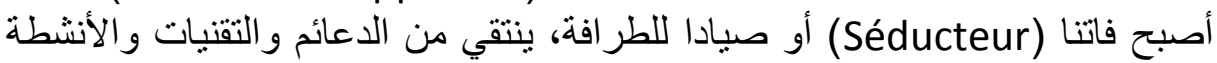

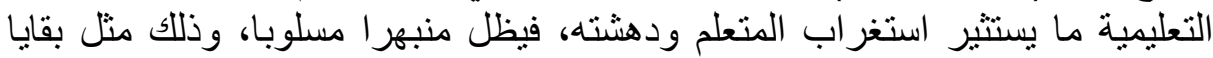

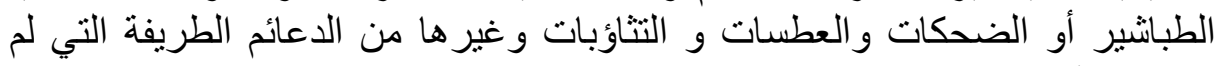
يسبق استثمار ها بيداغوجيا.

خاتـمة

لقد شهدت تعليمية اللغات الأجنبية تطورا مذهلا حرر المتعلم من قيود الفكر المتقارب La pensée ( (La pensée convergente)

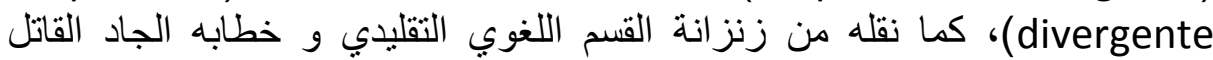

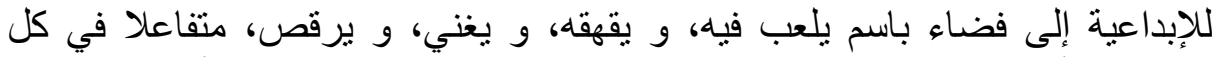
درس مع أنغام موسيقية و قصص مثيرة، و مكتشفا تقنيات ودعائم و أنشطة إبداعية فئائ

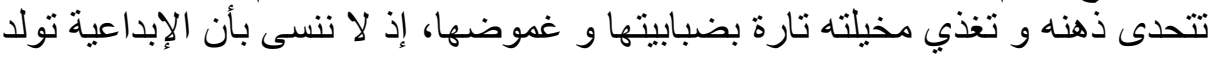

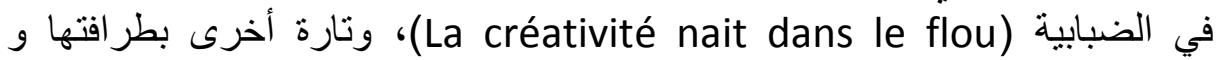
غر ابتها، و ذلك بمساعدة معلم "ساحر" منشط للضحك و الصدمة ) Promoteur du . (rire et du choc و هذا ما حاولنا تحقيقه مع طلبتنا كما بيناه عبر هذا المقال. 


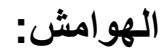

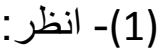

Richards.J, C \& platt and Chimdt, R. Dictionary of Language Teaching and Applied Linguistic, Longman, London, 3 Ed, 2002, P320.

(2)-Boiron,M. Approches pédagogiques de la chanson. Cavilam. Vichy

http://www.tv5momde.com/TV5Site/upload_image/app_ens_doc/ 26 fichier

approchechansons.pdf

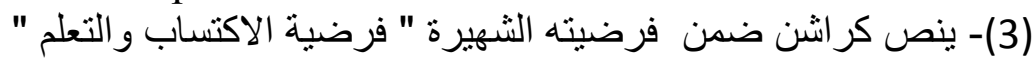

أنه في الوضع الاكتسابي تتسرب (The Acquisition-Learning Hypothesis)

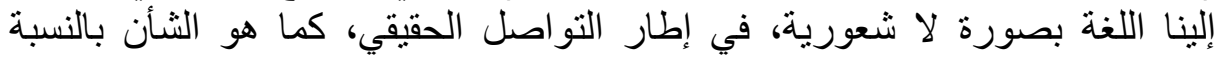

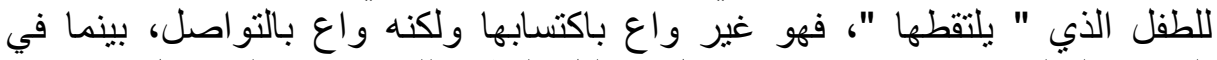

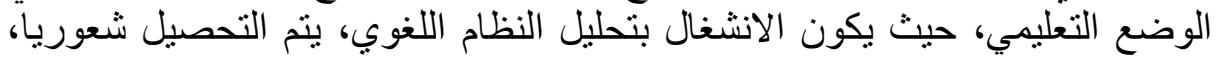
بمعزل عن التفاعل الحي. لمزيد من الاطلاع حول هذه الإن الفرضية، انظر:

Krashen, S.D and Tracy D. Terrell, the Natural Approach. Language acquisition in the classroom, Pergamon press, oxford, $2^{\text {ed }}$ Edition, 1985. P26.

(4)-Moskowitz.G. Connecting the powers of music to the learning of languages. The journal of the imagination in language learning and teaching, vol IV 1997.

http:/www.njcu/cill/vol4/adkins.html

(5)-Moskowitz.G. humanistic imagination. Soul food for language classroom. The journal of the imagination in language learning and teaching, vol II 1994.

http://www.njcu/cill/vol2/moskowitz.html

(6)-Eliason.W. The right to de be Creative. The journal of the imagination in language learning and teaching, vol I 1993.

http://www.njcu/cill/voll/eliason.html 